\title{
Optimizing a Hybrid Closed Loop System in Type 1 Diabetes: A Case Report
}

\author{
Goran Petrovski (D) - Fawziya Al Khalaf - Khalid Hussain • \\ Judith Campbell
}

Received: May 31, 2018 / Published online: July 20, 2018

(C) The Author(s) 2018

\begin{abstract}
We present the optimization of a hybrid closedloop (HCL) insulin pump system in a type 1 diabetes (T1D) patient in the first 3 months of using the Minimed 670G. An 11-year-old female with 7 years of T1D history started using the Minimed 670G HCL system at our center. The patient had been using the Medtronic Veo insulin pump without a sensor for almost 4 years with $\mathrm{HbA1c}$ levels between $7.8 \%$ and $10.2 \%$. We introduced the Minimed 670G to improve overall glucose control, improve quality of life and lessen the diabetes burden. Insulin pump characteristics of the Minimed Veo pump were used as the initial setting in the HCL system. The optimization of the Minimed 670G in the next 3 months led to the following changes: an insulin-to-carbohydrate ratio (ICHR) from 1 to $10 \mathrm{~g}$ in the morning period ( 6 a.m. to 12 a.m.) and $15-12 \mathrm{~g}$ for the rest of the day (12 p.m. to 6 a.m.); an active insulin time from 4 to $3 \mathrm{~h}$ and insulin sensitivity factor (ISF) from 120 to $90 \mathrm{mg} / \mathrm{dl}$ with no change in
\end{abstract}

Enhanced Digital Features To view enhanced digital features for this article, go to https://doi.org/10.6084/ m9.figshare.6798806.

G. Petrovski ( $)$ · F. Al Khalaf · K. Hussain ·

J. Campbell

Pediatric Endocrine and Diabetes Division, Sidra

Medicine, Doha, Qatar

e-mail: goran.endo@gmail.com target range. The HbA1c level decreased by $-1.3 \%$, and the time in range significantly increased to $77 \%$ with SG values of $139 \pm 60 \mathrm{mg} / \mathrm{dl}$, sensor wear of $82 \%$ and an auto mode period of $84 \%$ per week. Health providers should consider immediate adjustment of the bolus wizard settings such as the ICHR, ISF and active insulin time. The HCL system can improve overall glucose control with increased time in the range of sensor glucose values and reduction of HbA1c levels.

Keywords: Glucose control; Hybrid closed loop; Optimization; Type 1 diabetes

\section{INTRODUCTION}

Continuous subcutaneous insulin infusion (CSII) with continuous glucose monitoring (CGM) is one of the treatment options for type 1 diabetes (T1D) patients and can improve glucose control with fewer hypoglycemic events, decreased glycemic variability and improved quality of life. Technologic developments in diabetes have resulted in a substantial rise in the use of insulin pumps around the world.

Both academia and industry are currently developing artificial pancreas systems and closed-loop insulin delivery (CL) systems. The system uses a control algorithm to automatically increase, decrease or suspend insulin 
delivery using subcutaneous glucose sensor data to improve glucose control without increased exposure to hypoglycemia and to lessen the burden of diabetes management. One of the advantages of CL systems is the reduction in overall time spent in the hypoglycemic range. Automating the delivery of insulin in wearable systems combines three functions: continuous glucose monitoring, insulin delivery and control of insulin using specific algorithms.

Most of the CL systems under development are hybrid closed-loop (HCL) systems [1] where the patient is still required to estimate meal carbohydrate intake and confirm the recommended bolus insulin dose based on the bolus wizard setting. One of the available HCL systems is the Minimed 670G (Medtronic, Dublin, Ireland). When the HCL system is in "auto mode [2]," basal insulin delivery is adjusted by the subcutaneous glucose (SG) sensor every 5 min. Several studies reported that HCL systems $[3,4]$ could be used safely by adolescents and adults with type 1 diabetes in an outpatient setting, with no episodes of severe hypoglycemia or diabetic ketoacidosis. To date, the Minimed 670G HCL system is only used clinically in the USA.

In this case report, we present the optimization of the HCL system in a T1D patient in the first 3 months of using the Minimed 670G. To the best of our knowledge, this is the first patient with the HCL system to be reported in Qatar and the Middle East.

\section{CASE PRESENTATION}

An 11-year-old female with a 7-year T1D history started using the Minimed 670G HCL system at our center. The patient had been using a Medtronic Veo insulin pump without a sensor for almost 4 years. HbA1c levels were between $7.8 \%$ and $10.2 \%$ (last year), with no evidence of diabetic ketoacidosis or severe hypoglycemic events. Follow-up appointments were kept with a diabetologist, diabetes educator and registered dietitian on a regular 2-4-month basis.

All procedures performed in the study involving human participants were in accordance with the ethical standards of the institutional and/or national research committee and with the 1964 Helsinki Declaration and its later amendments or comparable ethical standards. Informed assent and consent were obtained from the patient and her caregiver.

Diabetes management on the Medtronic Veo insulin pump before transition showed the following results: HbA1c level 7.6\% (measured by Siemens DCA Vantage, Siemens AG, Munich, Germany); blood glucose (BG) values $198 \pm 118 \mathrm{mg} / \mathrm{dl}$; total daily insulin (TDI) $25.4 \pm 3.1$ units; basal ratio $45 \%$; total carbohydrate level $183 \pm 50 \mathrm{~g} /$ day; regular bolus wizard use (as shown in Fig. 1).

CSII characteristics prior to the upgrade were as follows: five basal rates with an average of 0.425 (minimum 0.400; maximum 0.525) units/ $\mathrm{h}$; insulin-to-carbohydrate ratio (ICHR) $15 \mathrm{~g}$; insulin sensitivity factor (ISF) $120 \mathrm{mg} / \mathrm{dl}$; target range 90-120 mg/dl; active insulin time $4 \mathrm{~h}$.

Both the patient's family and health care team agreed to upgrade to the Minimed 670G HCL system because of the huge glucose variability (BG $198 \pm 118 \mathrm{mg} / \mathrm{dl}$ ) and to improve overall glucose control, improve quality of life and lessen the diabetes burden.

Technical education on the HCL system with re-education on diabetes was performed in $5 \mathrm{~h}$ (three consequent sessions) in outpatient settings where the focus was on the CGM, calibration and auto mode function.

CSII characteristics from the Minimed Veo pump were used as the initial setting in the HCL system. Follow-up contacts were on a 1-week basis in the 1st month and a 2 -week period in the following 2 months. Most of the visits (75\%) were performed remotely using the phone, email or what's app, where the patient uploaded the HCL system at home and was asked to make changes according to the physician's advice.

Due to high post-prandial spikes, the ICHR was changed from 15 to $12 \mathrm{~g}$ in the $1 \mathrm{st}$ month, with an additional $12-10 \mathrm{~g}$ in the morning period ( 6 a.m. to 12 a.m.). The active insulin time was decreased from 4 to $3 \mathrm{~h}$.

Due to prolonged hyperglycemic spikes after bolus correction (BG > $200 \mathrm{mg} / \mathrm{dl}$ ), ISF was significantly decreased from 120 to $90 \mathrm{mg} / \mathrm{dl}$ with no change in the target range. We did not find a 
A

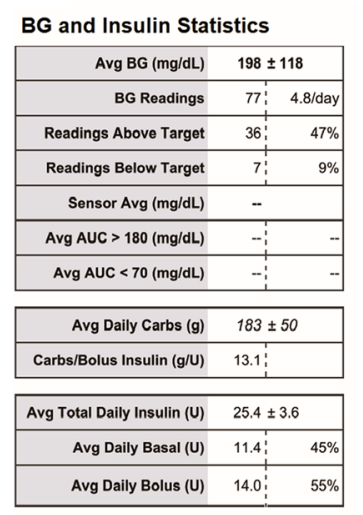

B

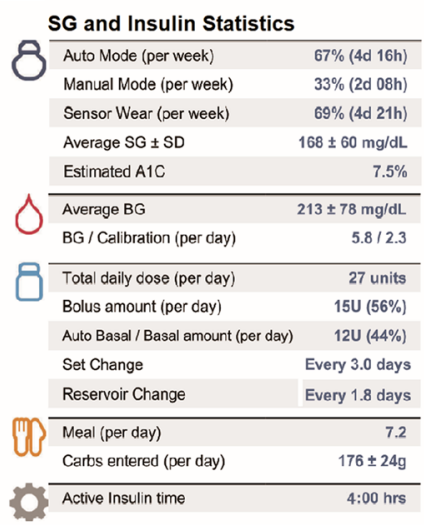

Fig. 1 The 24-h glucose profile on different CSII systems. Overall glucose control was improved (as shown in Fig. 2), where time in range $(70-180 \mathrm{mg} / \mathrm{dl})$ increased from $46 \%$ on the Minimed Veo Pump to $77 \%$, and the HbAlc level significantly decreased from $7.6 \%$ to $6.3 \%$ (both measured by Siemens DCA Vantage) in the following 3 months.

significant difference (as shown in Fig. 1) in the TDI dose ( $25.4 \pm 3.1$ vs. $27 \pm 1.8$ units), basal ratio ( $45 \%$ basal vs. $44 \%$ auto basal/basal amount) and total carbohydrate intake $(176 \pm 24$ vs. $184 \pm 36)$ per day. No diabetic ketoacidosis or severe hypoglycemic events were noted.

We achieved average SG values of $139 \pm 60 \mathrm{mg} / \mathrm{dl}$, sensor wear of $82 \%$ and auto mode period of $84 \%$ after 3 months (as shown in Fig. 1).

\section{DISCUSSION AND CONCLUSION}

Despite certain limitations, we have provided a general overview of the transition from conventional CSII to an HCL system and optimization of therapy in a T1D patient using the Minimed 670G at our institution. The teamwork of physicians, diabetes educators, dietitians and nurses achieved the main objective of improving the glucose control in our patient. Technical education was performed in the outpatient settings, and most of the follow-up contacts were remotely obtained, which allowed smooth transition to the HCL system.

The HbA1c level decreased by $-1.3 \%$, and time in range significantly increased to $77 \%$

\section{C}
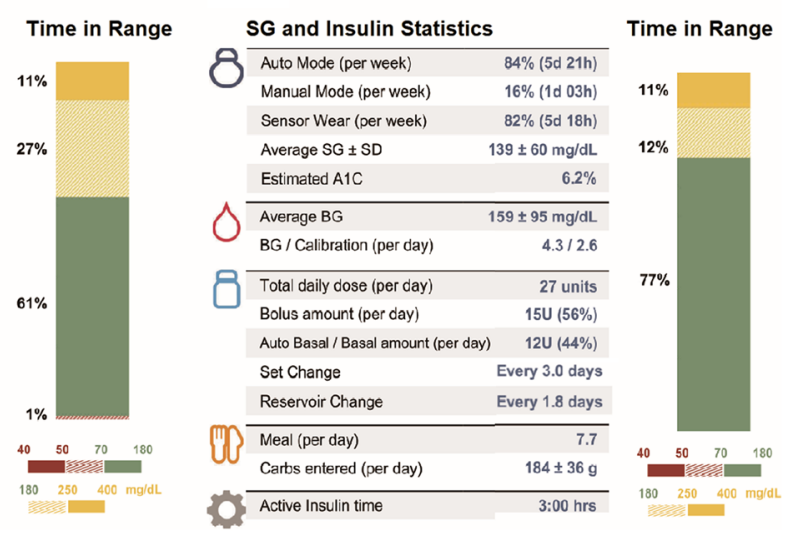

a Minimed Veo without sensor; b minimed 670G HCL system in the 1st week; c minimed 670G HCL system after 3 months. BG values, SG values and daily carbohydrates are expressed as mean \pm standard deviation unless otherwise indicated

with SG values of $139 \pm 60 \mathrm{mg} / \mathrm{dl}$. Possible reasons for improved glucose control were frequent contacts, patient motivation and diabetes team motivation. A recent meta-analysis [5] reported that current HCL systems should achieve at least $70 \%$ of sensor glucose values between 70 and $180 \mathrm{mg} / \mathrm{dl}$, with $<4 \%$ of values $<70 \mathrm{mg} / \mathrm{dl}$ and an SG average of $155 \mathrm{mg} / \mathrm{dl}$, equivalent to an estimated HbA1c of $7.0 \%$. Our patient achieved these metrics in a period of 3 months. One of the limitations of evaluating the time in range in this case report was having no CGM data on the previous insulin pump. However, the CGM data in the 1st week after the transition to the HCL system with the same settings can be considered a point for evaluation. The recent studies on HCL systems [3, 4] have also reported clinically important reductions in HbA1c levels and increases in the time in-target range while reducing the time below and above target levels.

We used the current settings from the Medtronic Veo pump while transitioning to the Minimed 670G, which directed us to adjust the bolus wizard settings (ICHR, ISF and active insulin time) several times to improve glucose control. Frequent contacts and a systematic approach to downloaded CSII data allowed us to 
A

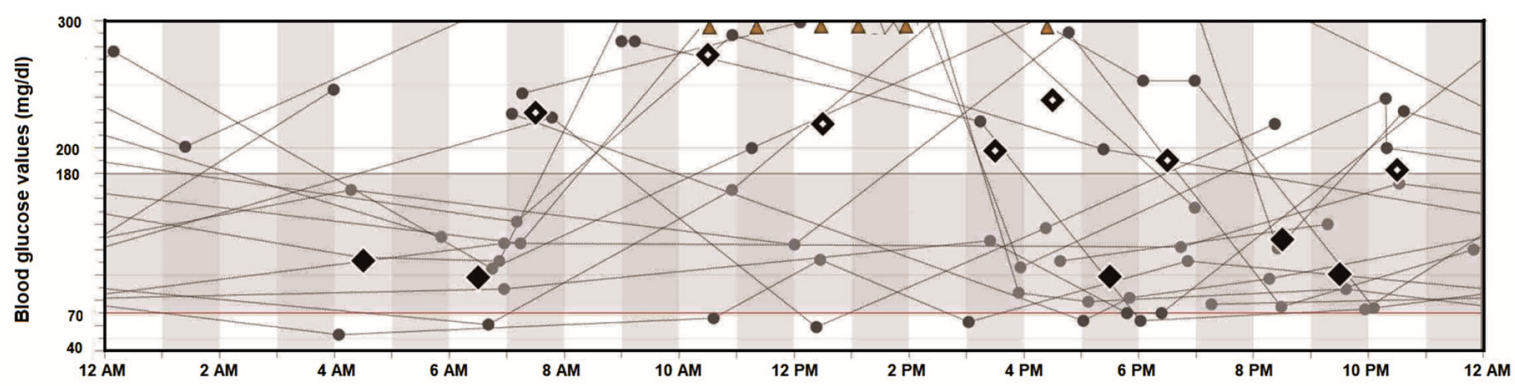

B

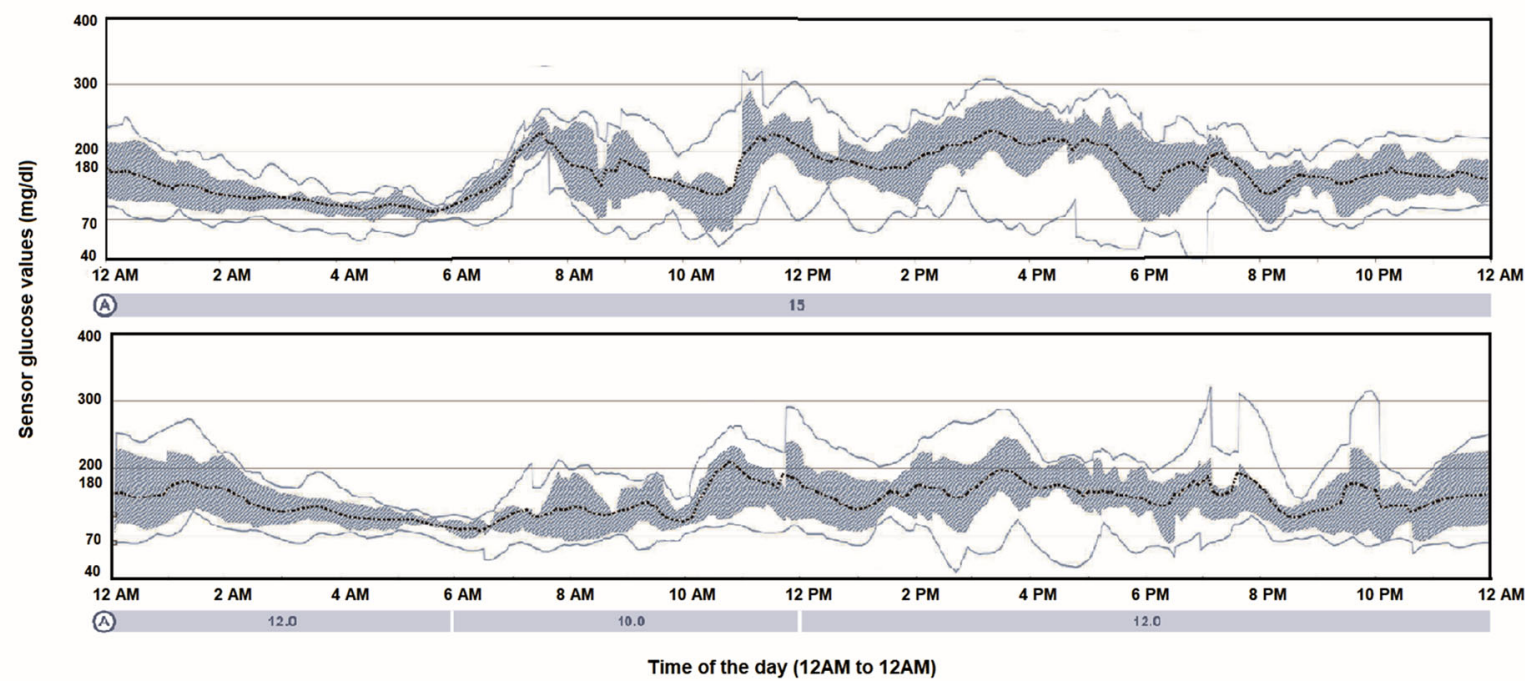

BG Graph: $\bullet$ BG Reading $\nabla \Delta$ Off Chart $\bullet$ Average with target range $\bullet$ Average outside target range

SG Graph: Percentile comparision $25-75 \% \quad \square 0-90 \quad \cdots \cdots \cdots . .$. Average

Fig. 2 Glucose and insulin statistics on different CSII systems. a Minimed Veo without sensor; b minimed 670G HCL system in the 1st week; c Minimed 670G HCL system after 3 months. BG graph: filled circle, BG reading;

fine-tune the CSII settings. Health care providers should anticipate immediate ICHR and active insulin time adjustments in young patients' transition from the conventional CSII to an HCL system.

We did not find any episode of severe hypoglycemia or diabetic ketoacidosis, which was also reported in a recent study [4].

Different CGM modalities (retrospective, real-time or flash monitoring) can be beneficial for improving the glucose control and increasing time in range in T1D patients. The ability of the integrated system to automatically and safely increase, decrease and suspend basal insulin delivery represents an important advance in T1D therapy for individuals with triangles, off chart; filled diamond, average within target range; diamond with white circle, average outside target range. SG graph: percentile comparison: filled square, 25-75\%; open square, 0-90\%; dotted line, average

diabetes, their families and health care teams. We believe the HCL system could be a transformative therapeutic option for T1D patients and their families.

Health care providers should consider immediate adjustment of the bolus wizard settings such as ICHR, ISF and active insulin time when switching from a conventional insulin pump to an HCL system. The HCL system with its features and good collaboration between health care staff and the patient and his/her caregiver can improve overall glucose control with increased time in range of sensor glucose values and reduction of HbA1c levels in T1D patients. 


\section{ACKNOWLEDGEMENTS}

We thank the patient and her family for participating in the study.

Funding. No funding or sponsorship was received for this study or publication of this article. The article processing charges were funded by the authors.

Authorship. All named authors meet the International Committee of Medical Journal Editors (ICMJE) criteria for authorship for this article, take responsibility for the integrity of the work as a whole and have given their approval for this version to be published.

Disclosures. Goran Petrovski, Fawziya Al Khalaf, Khalid Hussain and Judith Campbell have nothing to disclose.

Compliance with Ethics Guidelines. All procedures performed in studies involving human participants were in accordance with the ethical standards of the institutional and/or national research committee and with the 1964 Helsinki Declaration and its later amendments or comparable ethical standards. Informed assent and consent were obtained from the patient and her caregiver.

Data Availability. Data sharing is not applicable to this article as no data sets were generated or analyzed during the current study.

Open Access. This article is distributed under the terms of the Creative Commons
Attribution-NonCommercial 4.0 International License (http://creativecommons.org/licenses/ by-nc/4.0/), which permits any noncommercial use, distribution, and reproduction in any medium, provided you give appropriate credit to the original author(s) and the source, provide a link to the Creative Commons license, and indicate if changes were made.

\section{REFERENCES}

1. Trevitt S, Simpson S, Wood A. Artificial pancreas device systems for the closed-loop control of type 1 diabetes: what systems are in development? J Diabetes Sci Technol. 2016;10(2):714-23. https://doi.org/10. $1177 / 1932296815617968$.

2. U.S. Food and Drug Administration (2016) MiniMed 670G system. Summary of safety and effectiveness data (SSED). https://www.accessdata.fda.gov/cdrh docs/pdf16/P160017S017B.pdf. Accessed 20 April 2018.

3. Bergenstal RM, Garg S, Weinzimer SA, et al. Safety of a hybrid closed-loop insulin delivery system in patients with type 1 diabetes. JAMA. 2016;316:1407-8. https:// doi.org/10.1001/jama.2016.11708.

4. Thabit H, Tauschmann M, Allen JM, et al. Home use of an artificial beta cell in type 1 diabetes. N Engl J Med. 2015;373:2129-40. https://doi.org/10.1056/ NEJMoa1509351.

5. Weisman A, Bai JW, Cardinez M, et al. Effect of artificial pancreas systems on glycemic control in patients with type 1 diabetes: a systematic review and meta-analysis of outpatient randomized controlled trials. Lancet Diabetes Endocrinol. 2017;5:501-12. https://doi.org/10.1016/S2213-8587(17)30167-5. 